\title{
AN APPROXIMATE THEORY FOR ELASTIC WAVES PROPAGATED THROUGH A HETEROGENEOUS MEDIUM AND ITS APPLICATIONS
}

\author{
Tameshige TsukudA \\ Geophysical Institute, Faculty of Science, University of Tokyo, Tokyo
}

(Received January 25, 1973)

\begin{abstract}
A mathematical expression of elastic waves reflected due to slight heterogeneity of the medium is presented explicitly to the first order approximation in the case of one-dimensional problems. The approximate solution of elastic waves propagated through a heterogeneous medium is obtained by regarding the effect of the heterogeneity as that of equivalent dynamical sources scattered within the medium and by applying Green's function to the first-order approximate wave equation.

Comparison between the approximate refiected wave and the exact one shows the validity of the present theory.

The applications of the solution are found in such problems as attenuation of elastic waves passing through a heterogeneous medium and interpretation of reflected waves from a certain layered structure.

The finite difference method is applied to the wave equation with the aid of the present theory in order to demonstrate the nature of elastic waves propagated through a heterogeneous medium.
\end{abstract}

\section{Introduction}

Seismic waves are affected in their wave forms and amplitudes by scattering due to heterogeneity of the medium. Therefore, it is of considerable importance to study theoretically the behavior of scattering of elastic waves propagated through a heterogeneous elastic medium. The one-dimensional treatment of this problem concerned with in this paper may be useful for evaluating the effect of the heterogeneity on elastic waves and understanding the nature of scattered waves.

As ONDA (1965) pointed out, it is convenient to assume that the heterogeneous medium is sandwiched between homogeneous half spaces so that we can distinguish among incident, reflected and transmitted waves. Then, the medium behaves like a filter, the characteristic of which is namely the reflectivity or the transmissivity of the heterogeneous medium with finite width.

If the heterogeneous medium consists of homogeneous layers with different physical constants, the theoretical characteristic can be readily calculated against a given numerical model. Furthermore, since any structure can be sufficiently approximated with such layers, the numerical solutions of the characteristics of individual models are practically obtainable for a given frequency band (see FucHs 1968).

On the contrary, the structure of a certain heterogeneous medium has not yet 
been directly and uniquely determined from the frequency-dependent reflectivity or transmissivity of the medium; the trial-and-error method seems to be predominant in today's investigations. In other words, the inverse problem has not been solved statisfactorily.

In order to find out the relationship between a medium structure and its reflectivity and transmissivity, it is necessary to investigate the mechanism of the generation of reflected waves. From this point of view, the present paper deals with an approximate theory of one-dimensional elastic waves propagated through a slightly heterogeneous medium.

Previously, Yoshry ama (1960), Yoshiyama and ONDA (1962) investigated a similar problem concerning a structure with a sinusoidal fluctuation with special attention to attenuation of a propagated wave. Their theory is based on Mathieu's equation in the frequency domain. An extention of their method was published by ONDA (1964). In his paper, more general fluctuation is treated by decomposing the fluctuation of the medium into Fourier Components. The treatment of the above researches is quite strict, but the formulations of the results are rather complicated.

In the present paper, the author proposes another approach to this problem. The approximate solution of reflected waves given in this paper should contribute to understanding gross aspect of the effect of the heterogeneity on elastic waves and may also be applicable to practical problems. Some of the results of this paper will be compared with those of the above-mentioned researchers in later sections.

\section{Theory}

For brevity, let us assume the density is constant in the entire space. Then, the parameter of heterogeneity is only the velocity of an elastic wave. Heterogeneity is assumed to appear in the range,

$$
0 \leq x \leq L,
$$

where $L$ means the length of the heterogeneous medium. Let us describe the velocity distribution $V(x)$ as follows:

$$
\begin{aligned}
& V^{2}(x)=V_{0}^{2}(1+\varepsilon f(x)), \quad-\infty<x<\infty \\
& f(x) \equiv 0, \quad x<0 \text { or } x>L,
\end{aligned}
$$

where $V_{0}$ is the constant velocity defined in a homogeneous region and $\varepsilon f(x)$ denotes a heterogeneous structure. Furthermore, let us assume that the heterogeneity is so slight that the perturbation method can be applied to our problem.

Hence, we can put $\varepsilon$ and $f(x)$ as follows:

$$
\begin{aligned}
& 0<\varepsilon \ll 1 \\
& \operatorname{Max} .|f(x)|=1 .
\end{aligned}
$$

The equation of motion is

$$
\frac{\partial^{2}}{\partial t^{2}} u-\frac{\partial}{\partial x}\left(V^{2}(x) \frac{\partial}{\partial x} u\right)=0
$$


where $u$ stands for displacement or particle velocity or acceleration etc.. Substituting (2) into (4) we obtain

$$
\frac{\partial^{2} u}{\partial t^{2}}-V_{0}^{2} \frac{\partial^{2}}{\partial x^{2}} u=\varepsilon V_{0}^{2} f(x) \frac{\partial^{2}}{\partial x^{2}} u+\varepsilon V_{0}^{2} f^{\prime}(x) \frac{\partial}{\partial x} u
$$

where

$$
f^{\prime}(x) \equiv \frac{d}{d x} f(x)
$$

In order to solve the partial differential Eq. (5), let us apply the perturbation method expanding $u$ as follows:

$$
\begin{aligned}
& u(x, t)=u_{0}+u_{1}+u_{2}+\cdots \\
& \left|u_{i}\right|=0\left(\varepsilon^{i}\right), \quad i=0,1,2,3, \cdots,
\end{aligned}
$$

where $u_{i}$ denotes the $i$-th-order approximate solution. Substituting expression (6) into Eq. (5), the following partial differential equations are obtained by equating the same order terms of $\varepsilon$ on the left- and right-hand sides of the equation.

$$
\begin{gathered}
\frac{\partial^{2}}{\partial t^{2}} u_{0}-V_{0}^{2} \frac{\partial^{2}}{\partial x^{2}} u_{0}=0, \\
\frac{\partial^{2}}{\partial t^{2}} u_{i}-V_{0}^{2} \frac{\partial^{2}}{\partial x^{2}} u_{i}=\varepsilon V_{0}^{2} f(x) \frac{\partial^{2}}{\partial x^{2}} u_{i-1}+\varepsilon V_{0}^{2} f^{\prime}(x) \frac{\partial}{\partial x} u_{i-1} \quad i=1,2,3, \cdots .
\end{gathered}
$$

The solution $u_{i}$ of Eq. (8) means a wave in a homogeneous medium $V=V_{0}$, excited by an exerted force proportional to the right-hand side of Eq. (8). The wave form of the zeroth-order approximate solution $u_{0}$, satisfying Eq. (7), can be given arbitrarily. Once $u_{0}$ is determined, the first-order approximate solution $u_{1}$, statisfying Eq. (8) for $i=1$, the right-hand side of which is described in terms of $u_{0}$, can be calculated by the use of the Green's function. The second-order approximate solution $u_{2}$ is derived from Eq. (8) for $i=2$. $u_{2}, u_{4}, \cdots$ are determined successively in the same way.

Let us take $u_{0}$ as

$$
u_{0}=\delta\left(x-V_{0} t\right),
$$

where $\delta(x)$ denotes the Dirac's delta function. This impulsive wave, which we take as an incident wave to the heterogeneous region, in propagated with the velocity $V_{0}$ of the homogeneous medium through the entire space passing $x=0$ at $t=0$.

From Eqs. (8) and (9), the first-order approximate solution $u_{1}$ should statisfy the following equation

$$
\frac{\partial^{2}}{\partial t^{2}} u_{1}-V_{0}^{2} \frac{\partial^{2}}{\partial x^{2}} u_{1}=\varepsilon V_{0}^{2} f(x) \delta^{\prime \prime}\left(x-V_{0} t\right)+\varepsilon V_{0}^{2} f^{\prime}(x) \delta^{\prime}\left(x-V_{0} t\right) .
$$

In order to solve the wave Eq. (10), the Green's function $G_{0}(x, t ; \xi, \tau)$ is introduced, which satisfies the inhomogeneous partial differential equation

$$
\frac{\partial^{2}}{\partial t^{2}} G_{0}(x, t ; \xi, \tau)-V_{0}^{2} \frac{\partial^{2}}{\partial x^{2}} G_{0}(x, t ; \xi, \tau)=V_{0}^{2} \delta(x-\xi) \delta(t-\tau) \text {. }
$$


The solution of Eq. (11) is well known in mathematical physics. That is

$$
G_{0}(x, t ; \xi, \tau)=\left\{\begin{array}{l}
0\left\{\begin{array}{l}
t<\tau \\
\tau<t, \quad|x-\xi|>V_{0}|t-\tau|
\end{array}\right. \\
\frac{V_{0}}{2}, \quad \tau<t, \quad|x-\xi|<V_{0}|t-\tau| .
\end{array}\right.
$$

In terms of the Green's function, the solution of the equation

$$
\frac{\partial^{2}}{\partial t^{2}} u-V_{0}^{2} \frac{\partial^{2}}{\partial x^{2}} u=V_{0}^{2} g(x, t),
$$

is obtained as

$$
u=\int_{-\infty}^{\infty} \int_{-\infty}^{\infty} G_{0}(x, t ; \xi, \tau) g(\xi, \tau) d \xi d \tau
$$

In our case, the above $g(\xi, \tau)$ takes the form

$$
g(\xi, \tau)=\varepsilon f(\xi) \delta^{\prime \prime}\left(\xi-V_{0} \tau\right)+\varepsilon f^{\prime}(\hat{\xi}) \delta^{\prime}\left(\xi-V_{0} \tau\right) .
$$

Therefore, the solution of Eq. (10) is given as

$$
u_{1}=v_{1}+v_{2}
$$

where,

$$
\begin{aligned}
& v_{1}=\varepsilon \int_{-\infty}^{\infty} \int_{-\infty}^{\infty} G_{0}(x, t ; \xi, \tau) f(\xi) \delta^{\prime \prime}\left(\xi-V_{0} \tau\right) \mathrm{d} \xi d \tau, \\
& v_{2}=\varepsilon \int_{-\infty}^{\infty} \int_{-\infty}^{\infty} G_{0}(x, t ; \xi, \tau) f^{\prime}(\xi) \delta^{\prime}\left(\varepsilon-V_{0} \tau\right) d \xi d \tau .
\end{aligned}
$$

Integrating by parts and taking account of $f(+\infty)=0$ and $f(-\infty)=0$, we can obtain $v_{1}$ as follows:

$$
\begin{aligned}
v_{1}= & \left.\varepsilon \int_{-\infty}^{\infty}\left[G_{0}(x, t ; \xi, \tau) f(\xi) \delta^{\prime}\left(\xi-V_{0} \tau\right)\right]\right|_{-\infty} ^{\infty} d \tau \\
& -\varepsilon \int_{-\infty}^{\infty} \int_{-\infty}^{\infty} \frac{\partial}{\partial \xi}\left\{G_{0}(x, t ; \xi, \tau) f(\xi)\right\} \delta^{\prime}\left(\xi-V_{0} \tau\right) d \xi d \tau \\
= & -\left.\varepsilon \int_{-\infty}^{\infty}\left[\frac{\partial}{\partial \xi}\left\{G_{0}(x, t ; \xi, \tau) f(\xi)\right\} \delta\left(\xi-V_{0} \tau\right)\right]\right|_{-\infty} ^{\infty} d \tau \\
& +\varepsilon \int_{-\infty}^{\infty} \int_{-\infty}^{\infty} \frac{\partial^{2}}{\partial \xi^{2}}\left\{G_{0}(x, t ; \xi, \tau) f(\xi)\right\} \delta\left(\xi-V_{0} \tau\right) d \xi d \tau \\
& =\varepsilon \int_{-\infty}^{\infty}\left[\frac{\partial^{2}}{\partial \xi^{2}}\left\{G_{0}(x, t ; \xi, \tau) f(\xi)\right\}\right]_{\xi=V_{0}=} d \tau
\end{aligned}
$$

By the way, from Eq. (12) we obtain the following relations:

$$
\frac{\partial}{\partial \xi} G_{0}(x, t ; \xi, \tau)=\frac{V_{0}}{2} \delta\left(\xi-x+V_{0} t-V_{0} \tau\right)-\frac{V_{0}}{2} \delta\left(\xi-x-V_{0} t+V_{0} \tau\right) \text {. }
$$




$$
\frac{\partial^{2}}{\partial \xi^{2}} G_{0}(x, t ; \xi, \tau)=\frac{V_{0}}{2} \delta^{\prime}\left(\xi-x+V_{0} t-V_{0} \tau\right)-\frac{V_{0}}{2} \delta^{\prime}\left(\xi-x-V_{0} t+V_{0} \tau\right)
$$

Substituting (18) and $(18)^{\prime}$ into (17) we get the final result of $v_{1}$ as follows,

$$
\begin{aligned}
v_{1}= & -\frac{\varepsilon}{2} \delta^{\prime}\left(x-V_{0} t\right) \int_{0}^{V_{0} t} f(\xi) d \xi-\varepsilon \delta\left(x-V_{0} t\right) \int_{0}^{V_{0} t} f^{\prime}(\xi) d \xi \\
& +\frac{\varepsilon}{8} f^{\prime}\left(\frac{x+V_{0} t}{2}\right)-\frac{\xi}{2} f^{\prime}(0), \quad-V_{0} t<x<V_{0} t .
\end{aligned}
$$

In the same way, we get

$$
\begin{aligned}
v_{2}= & \frac{\varepsilon}{2} \delta\left(x-V_{0} t\right) \int_{0}^{V_{0} t} f^{\prime}(\xi) d \xi-\frac{\varepsilon}{4} f^{\prime}\left(\frac{x+V_{0} t}{2}\right) \\
& +\frac{\varepsilon}{2} f^{\prime}(0), \quad-V_{0} t<x<V_{0} t .
\end{aligned}
$$

Eventually, the solution of Eq. (10) is

$$
\begin{aligned}
u_{1}= & -\frac{\varepsilon}{2}\left[\delta^{\prime}\left(x-V_{0} t\right) \int_{0}^{V_{0} t} f(\xi) d \xi+\delta\left(x-V_{0} t\right) \int_{0}^{V_{0} t} f^{\prime}(\xi) d \xi\right] \\
& -\frac{\varepsilon}{8} f^{\prime}\left(\frac{x+V_{0} t}{2}\right), \quad-V_{0} t<x<V_{0} t .
\end{aligned}
$$

The higher order approximate solutions than above treated have not simple forms, because it is impossible to carry out such an integration as in (17). So that, the formulation is limited to the first-order approximation in this paper. However, it should be stressed that the simplicity of the result is the merit of the present method, although the evaluation of the higher order value is difficult in this theoretical method. The solution (21) is considered to be an impulse response of the heterogenous medium. For an arbitrary form of the incident wave

$$
u_{0}= \begin{cases}w\left(x-V_{0} t\right), & x-V_{0} t \leq 0 \\ 0, & x-V_{0} t>0,\end{cases}
$$

the first-order approximate solution is

$$
u_{0_{1}}=\int_{-\infty}^{0} G(x, t ; \xi) w(\xi) d \xi
$$

where,

$$
\begin{aligned}
G(x, t ; \xi)= & -\frac{\varepsilon}{2}\left[\delta^{\prime}\left(x-\xi-V_{0} t\right) \int_{0}^{V_{0} t} f(\xi) d \xi+\delta\left(x-\xi-V_{0} t\right) \int_{0}^{V_{0} t} f^{\prime}(\xi) d \xi\right] \\
& -\frac{\varepsilon}{8} f^{\prime}\left(\frac{x-\xi+V_{0} t}{2}\right), \quad-V_{0} t<x-\xi<V_{0} t .
\end{aligned}
$$

Let us go back to the solution (21). The last term means a reflected wave. We can calculate the wave form by differentiating the structural function $f(x)$, lengthening its wavelength two times, multiplying it by $\varepsilon / 8$ and changing its sign. 
The other terms contribute to the disturbance of the transmitted wave. If the following conditions

$$
\begin{aligned}
& \int_{0}^{V_{0} t} f(\xi) d \xi=0(\varepsilon) \\
& \int_{0}^{V_{0} t} f^{\prime}(\xi) d \xi=0(\varepsilon)
\end{aligned}
$$

hold, we can neglect such terms so far as the first-order approximation is concerned. The above conditions require the heterogeneity should have an oscillatory structure.

Taking the conditions (25) into account, the results of the above approximate theory can be summarized as follows:

(i) The transmitted wave is not disturbed by the heterogeneity in its wave form and the propagation velocity, in so far as the first order approximation is concerned; the disturbance may be found in the higher order solutions. Therefore, the dispersion of the propagation velocity of a transmitted wave does not occur in this approximation. These results are also found in the paper of ONDA (1964).

(ii) The reflected wave can be easily obtained as already mentioned. It is concluded that the amplitude of the reflectivity of a heterogeneous medium is the order $\varepsilon$; its dependence on the wavelength (or frequency) is such that the intensive reflection occurs, when the wavelength of the incident wave is two times of the prominent structural wave length of the heterogeneous medium. Figure 1 illustrates the above nature; the incident impulse is propagated in the right direction through the heterogeneous medium (in the figure, the fluctuation of the structure is as follows,

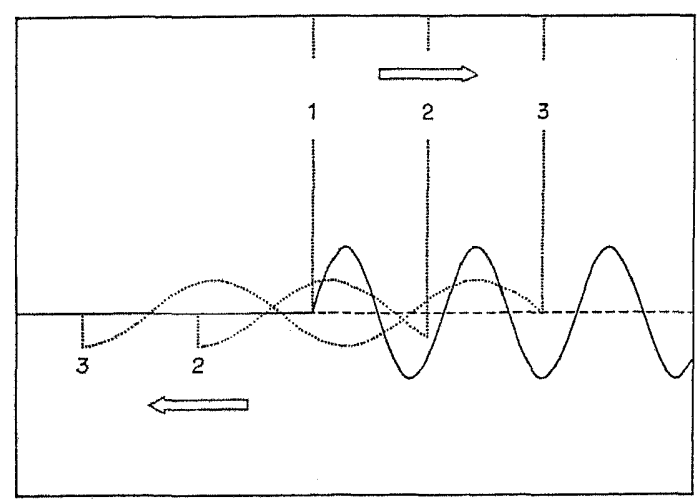

Fig. 1. Illustration of the nature of an elastic wave incident to a heterogeneous medium with a certain sinusoidal fluctuation.

$$
f(x)= \begin{cases}\sin \left(\frac{2 \pi}{\lambda_{s}} \cdot x\right), & x \geq 0 \\ 0, & x<0,\end{cases}
$$

where $\lambda_{g}$ denotes the wavelength of the structure); the reflected wave is generated 
and is propagated in the left direction extending the length of its wave train in accordance with the propagation of the incident pulse.

If we take density as a parameter of a heterogeneous medium, the formulation of the equation of motion should be somewhat altered from Eq. (4) and the structure of the medium is described by the distributions of density and elastic constant rather than that of velocity as given in (2). Let the distributions of density $\rho$ and elastic constant $E$ be

$$
\left.\left.\begin{array}{l}
\rho(x)=\rho_{0}\left(1+\varepsilon_{p} g(x)\right) \\
E(x)=E_{0}\left(1+\varepsilon_{E} h(x)\right)
\end{array}\right\}-\infty<x<\infty \quad\right\}
$$

The equation of motion corresponding to (4) is

$$
\rho(x) \frac{\partial^{2}}{\partial t^{2}} u-\frac{\partial}{\partial x}\left(E(x) \frac{\partial}{\partial x} u\right)=0 .
$$

Substituting expression (27) into Eq. (28) and neglecting the higher order terms than $0(\varepsilon)$, Eq. (28) yields

$$
\frac{\partial^{2}}{\partial t^{2}} u-V_{0}^{2} \frac{\partial^{2}}{\partial x^{2}} u=\varepsilon_{E} V_{0}^{2} h(x) \frac{\partial^{2}}{\partial x^{2}} u+\varepsilon_{E} V_{0}^{2} h^{\prime}(x) \frac{\partial}{\partial x} u-\varepsilon_{\rho}^{\Perp} V_{0}^{2} g(x) \frac{\partial^{2}}{\partial x^{2}} u,
$$

where $V_{0}=\sqrt{E_{0} / \rho_{0}}$, the velocity in the homogeneous region of the medium. From Eq. (29) we can get the first-order approximate solution corresponding to (21). The reflected wave in accordance with the incident wave represented as (9) is

$$
u_{\mathrm{ret} .}=-\frac{\varepsilon_{E}}{8} h^{\prime}\left(\frac{x+V_{0} t}{2}\right)-\frac{\varepsilon_{\rho}}{8} g^{\prime}\left(\frac{x+V_{0} t}{2}\right)
$$

The accoustic impedance of the medium is defined to be $\sqrt{\rho E}$, whose distribution is obtained as

$$
\sqrt{\rho E}=\sqrt{\rho_{0} E_{0}}\left(1+\varepsilon_{E} h(x)+\varepsilon_{o} g(x)\right),
$$

to the first order approximation. When the accoustic impedance is constant in the whole medium, from (31) following relation holds.

$$
\varepsilon_{E} h(x)+\varepsilon_{\rho} g(x)=0, \quad-\infty<x<\infty .
$$

Differentiating both sides of Eq. (32) we obtain

$$
\varepsilon_{E} h^{\prime}(x)+\varepsilon_{p} g^{\prime}(x)=0, \quad-\infty<x<\infty .
$$

In this case the right-hand side of (30) vanishes and the reflection does not occur.

In later sections the treatment is restricted to the case of constant density, but the more general consideration can be readily made as explained in the last part of the present section. 


\section{Examination of the Present Theory}

In order to confirm the validity of the present theory, let us try to examine the differences between the two reflectivities: the one is the approximate reflectivity obtained in the present theory; the other is nearly exact one, which is computed by the use of the method of a propagator matrix for an approximate multi-layered model of a given heterogeneous medium.

The test models are sinusoidally fluctuated medium and a layered medium with a periodic structure. The form of the latter structure contains intensively higherorder Fourier components of the fluctuation. Therefore, this case serves as an example of rather complex structures. Furthermore, for this layered structure, the matrix method gives us exact solutions of the reflectivities.

At the beginning of the examination, let us make a comparison between the spectrum of the reflected waves calculated by the use of the present theory and the matrix method above mentioned in the case of the one-cycle structure of the medium. For a following sinusoidal structure,

$$
f(x)= \begin{cases}-\sin \left(\frac{2 \pi}{\lambda_{s}} \cdot x\right), & 0 \leq x \leq \lambda_{s} \\ 0, & x<0 \text { or } x>\lambda_{s},\end{cases}
$$

The term of the reflected wave in the right-hand side of (21) yields

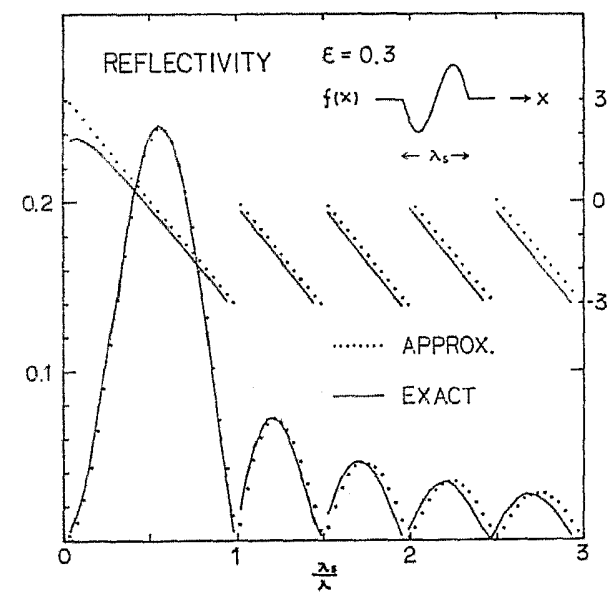

Fig. 2. Amplitude and phase angle of the reflectivity versus $\lambda_{s} / \lambda$ obtained by both the present theory (dotted line) and the matrix method (solid line) for $\varepsilon=0.3$ and

$$
f(x)=\left\{\begin{array}{cl}
-\sin \left(\frac{2 \pi}{\lambda_{s}} \cdot x\right) & \left(0 \leq x \leq \lambda_{s}\right) \\
0 & \left(x<0 \text { or } x>\lambda_{s}\right) .
\end{array}\right.
$$

In the matrix method the medium is divided into 40 layers per one cycle structure. 


$$
u_{\mathrm{ref} .}= \begin{cases}\frac{\varepsilon}{8} \cdot \frac{2 \pi}{\lambda_{s}} \cdot \cos \left(\frac{\pi}{\lambda_{s}}\left(x+V_{0} t\right)\right), & -V_{0} t<x<\operatorname{Min} .\left(V_{0} t, L-V_{0} t\right) \\ 0, & \text { elsewhere. }\end{cases}
$$

the reflectivity of the medium defined as (34) is equivalent to the spectral ratio of $u_{r \in f}(0, t)$ to $\delta\left(-V_{0} t\right)$, which is readily obtained theoretically as

$$
R(\omega)=\frac{\varepsilon}{2} \cdot \frac{\omega_{0}}{2} \cdot \sin \left(\frac{\omega}{2} \cdot T\right) \cdot \frac{\omega / 2}{(\omega / 2)^{2}-\left(\omega_{0} / 2\right)^{2}} \cdot e^{-i \frac{\omega}{2} \cdot T},
$$

where, $\omega$ is angular frequency, $T=2 \lambda_{s} / V_{0}$ and $\omega_{0}=2 \pi / T$.

In Fig. 2, the amplitude and the phase characteristics of the reflectivity versus non-dimensional frequency $\lambda_{s} / \lambda$ are shown in the case of the sinusoidal fluctuation with one cycle as (34) for $\varepsilon=0.3$, which is a considerably large value in practical point of view and corresponds to about $14 \%$ in the velocity fluctuation. The amplitude characteristic of the present theory coincides well with the exact one around the main lobe of the spectrum. In the case of the phase characteristic the agreement is not so good as the amplitude. The imperfection of the phase characteristic of the present approximate theory will be made clearer in Section 5.

For the following periodic layered structure

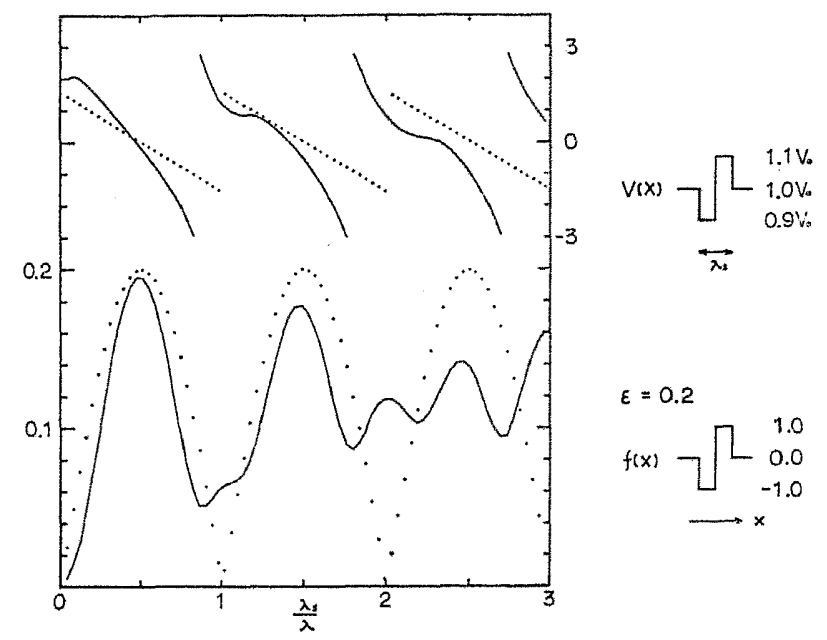

Fig. 3. Amplitude and phase angle of the reflectivity versus $\lambda_{s} / 2$ obtained by the present theory (dotted line) for $\varepsilon=0.2$ and

$$
f(x)=\left\{\begin{aligned}
-1 & \left(0<x<\lambda_{8} / 2\right) \\
1 & \left(\lambda_{s} / 2<x<\lambda_{s}\right) \\
0 & \left(x<0 \text { or } x>\lambda_{8}\right),
\end{aligned}\right.
$$

and by the matrix method (solid line) for

$$
\dot{V}(x)= \begin{cases}0.9 V_{0} & \left(0<x<\lambda_{s} / 2\right) \\ 1.1 V_{0} & \left(\lambda_{8} / 2<x<\lambda_{8}\right) \\ V_{0} & \left(x<0 \text { or } x>\lambda_{8}\right)\end{cases}
$$




$$
f(x)=\left\{\begin{array}{cc}
-1, & 0<x<\lambda_{B} / 2 \\
1, & \lambda_{8} / 2<x<\lambda_{8} \\
0, & \text { elsewhere }
\end{array}\right.
$$

the reflected wave is given by the formula

$$
u_{\mathrm{ref} .}=\frac{\varepsilon}{8}\left[\delta\left(\frac{x+V_{0} t}{2}\right)-2 \cdot \delta\left(\frac{x+V_{0} t}{2}-\frac{\lambda_{s}}{2}\right)+\delta\left(\frac{x+V_{0} t}{2}-\lambda_{s}\right)\right],
$$

and the reflectivity is formulated as

$$
R(\omega)=\frac{\varepsilon}{4}\left[1-2 e^{-i \frac{\omega}{2} \cdot T}+e^{-i \omega \cdot T}\right],
$$

where notations are the same as in (36).

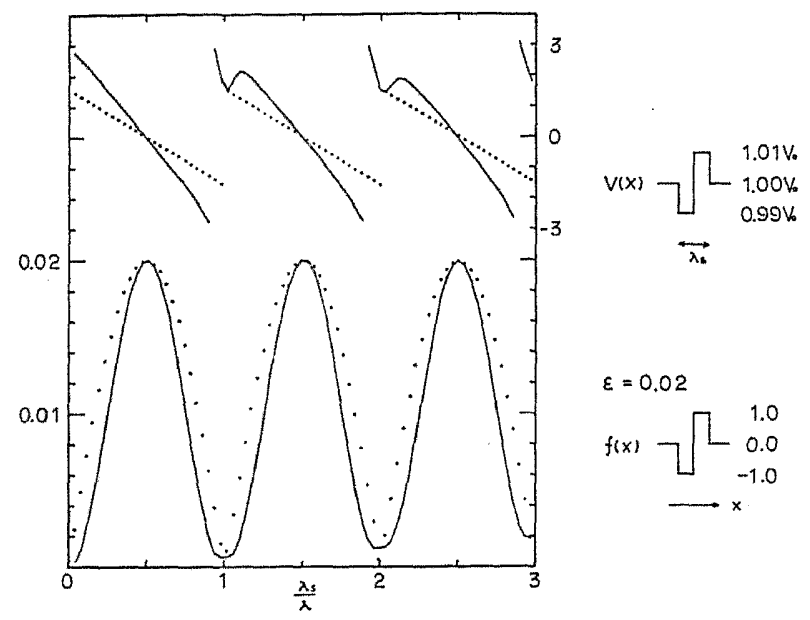

Fig. 4. Amplitude and phase angle of the reflectivity versus $\lambda_{8} / \lambda$ obtained by the present theory (dotted line) for $\varepsilon=0.02$ and

$$
f(x)=\left\{\begin{aligned}
-1 & \left(0<x<\lambda_{8} / 2\right) \\
1 & \left(\lambda_{8} / 2<x<\lambda_{8}\right) \\
0 & \left(x<0 \text { or } x>\lambda_{8}\right),
\end{aligned}\right.
$$

and by the matrix method (solid line) for

$$
V(x)=\left\{\begin{aligned}
0.99 V_{0} & \left(0<x<\lambda_{8} / 2\right) \\
1.01 V_{0} & \left(\lambda_{B} / 2<x<\lambda_{8}\right) \\
V_{0} & \left(x<0 \text { or } x>\lambda_{8}\right) .
\end{aligned}\right.
$$

Figures 3 and 4 show the amplitude and phase characteristics of the reflectivity versus $\lambda_{s} / \lambda$ in the case of a periodic layered structure for large and small amount of fluctuations respectively. The values indicated with solid lines are calculated exactly by the matrix method against the velocity structures as shown in the upper pictures in the right parts of Fig. 3 and Fig. 4. On the other hand the theoretical reflectivities given by (39) are based on the structure (37) and the values of $\varepsilon$ are designated as 
0.2 and 0.02 in Fig. 3 and Fig. 4, respectively.

We can see the behavior of the reflectivity of a thicker heterogeneous medium than one cycle in Fig. 5 and Fig. 6 computed by the matrix method for a sinusoidal fluctuation and a periodic layered structure respectively. The shape of the reflectivity does not change greatly with the thickness of the heterogeneous medium, whereas the amplitudes of the peaks grow with the thickness of the heterogeneous medium. Therefore, it may be sufficient to examine only the peak value of the amplitude characteristic of the reflectivity obtained by the approximate theory.

Let $L$ be the thickness of the heterogeneous medium, and put $L$ as

$$
L=n \lambda_{s},
$$

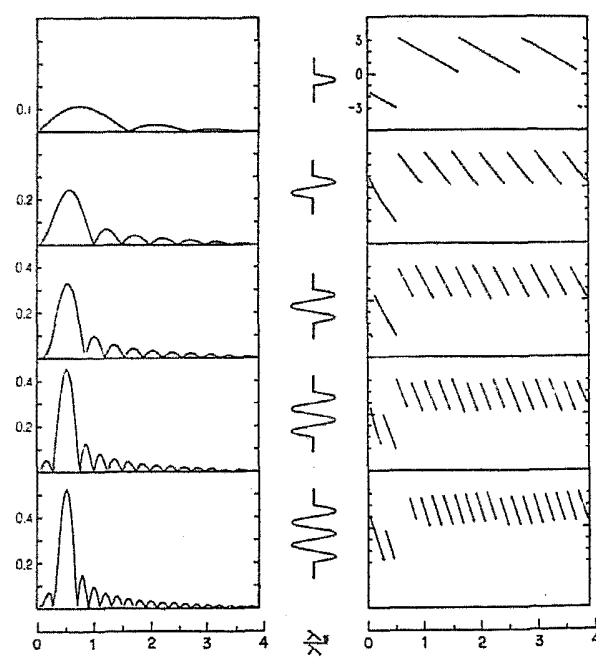

Fig. 5. Amplitude and phase characteristics of the reflectivities obtained by the matrix method for the layered models of sinusoidal structures for $\varepsilon=0.3$. The medium is divided into 40 layers per one cycle structure.

The structures given in the flgure are described so that the incident waves enter into the heterogeneous structures from the upper direction.
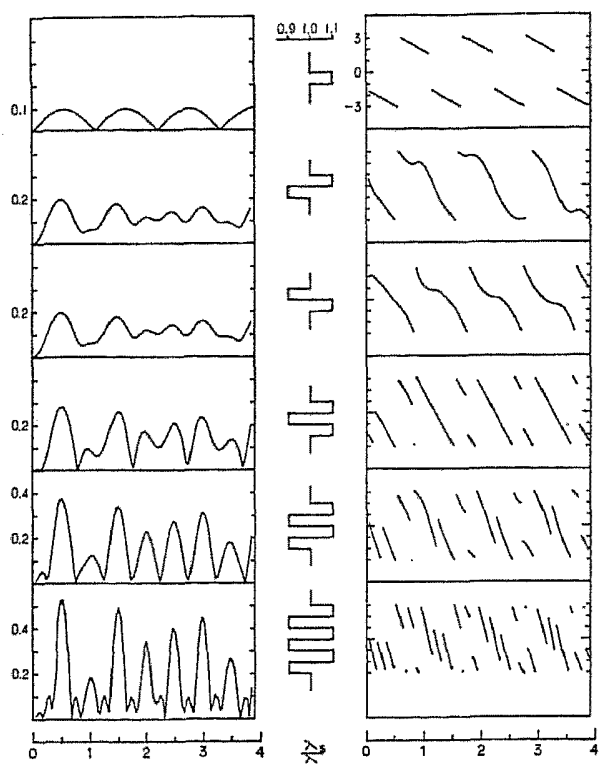

Fig. 6. Amplitude and phase characteristics of the reflectivities obtained by the matrix method for periodic layered structures.

The structures given in the figure are described so that the incident waves enter into the heterogeneous structures from the upper direction.

where $n=1 / 2,1,3 / 2, \cdots$.

The periodic structure is defined as follows:

$$
f(x)=\left\{\begin{array}{cl}
f_{0}\left(x-m \frac{\lambda_{s}}{2}\right) \cdot e^{i m \pi}, & \frac{m \lambda_{s}}{2} \leq x \leq \frac{(m+1) \lambda_{s}}{2} \\
(m=0,1,2, \cdots, 2 n-1) & \text { elsewhere }
\end{array}\right\}
$$


Table 1. Peak values of the amplitude characteristics of the reflectivities of sinusoidal structures for $\varepsilon=0.3$.

\begin{tabular}{ccc}
\hline \multicolumn{3}{c}{$\left|R_{n}\left(\omega_{0}\right)\right|$ (Sinusoidal Structure) } \\
\hline$n\left(=L / \lambda_{8}\right)$ & Matrix Method & Approx. Theory \\
\hline 0.5 & 0.099 & 0.118 \\
1 & 0.240 & 0.236 \\
1.5 & 0.331 & 0.354 \\
2 & 0.452 & 0.471 \\
2.5 & 0.528 & 0.589 \\
3 & 0.624 & 0.707 \\
\hline
\end{tabular}

Table 2. Peak values of the amplitude characteristics of the reflectivities of periodic layered structures.

In the matrix method the velocity structures are taken as

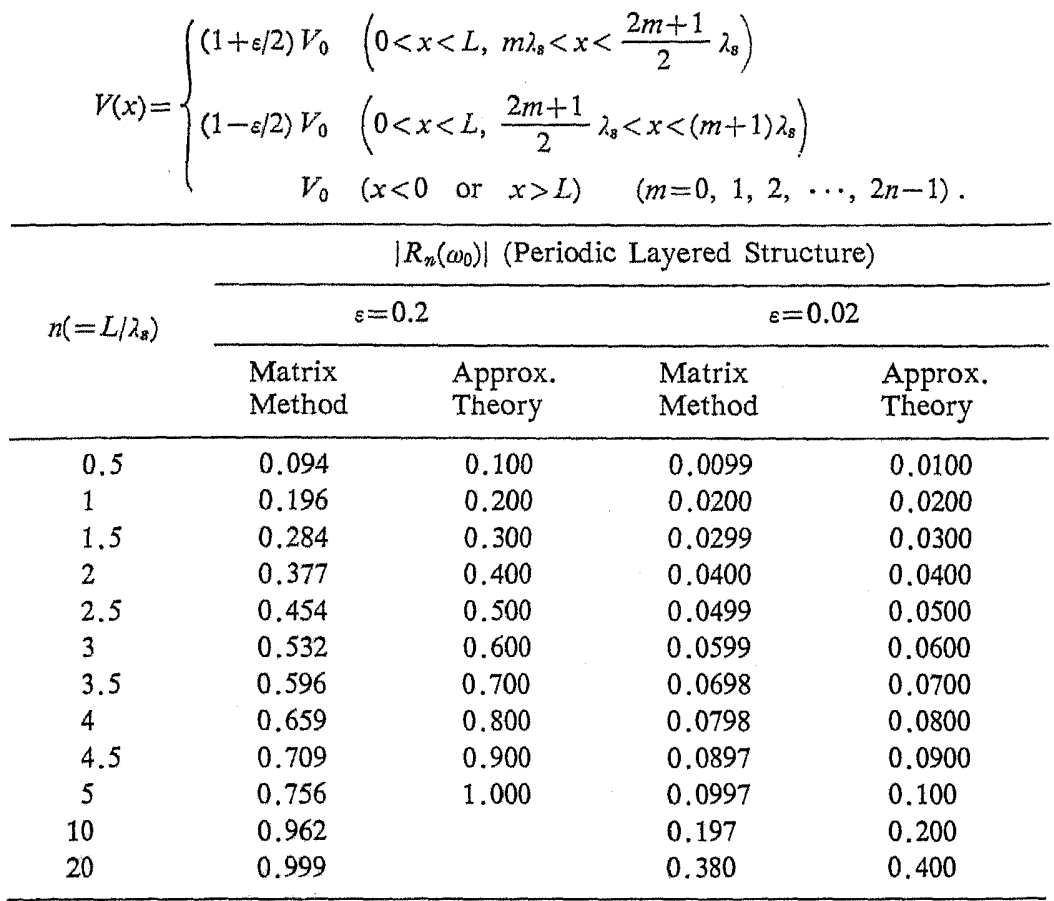

The associated reflceted wave observed at $x=0$ is

$$
u_{\text {raf } .}(t)=-\frac{\varepsilon}{8} \sum_{m=0}^{2 n-1} f_{0}^{\prime}\left(\frac{1}{2} V_{0} t-m \frac{\lambda_{s}}{2}\right) \cdot e^{i m \pi} .
$$

The Fourier transform of (42) should be written as

$$
U(\omega)=\sum_{m=0}^{2 n-1} F_{0}(\omega) e^{-i m \frac{\omega}{2} \cdot T} \cdot e^{i m \pi},
$$


where,

$$
F_{0}(\omega)=\int_{-\infty}^{\infty} f_{0}^{\prime}\left(\frac{1}{2} V_{0} t\right) e^{-i \omega t} d t
$$

and notations are the same as in (36). From Eq. (43), we get $U(\omega)$ at $\omega=\omega_{0} \equiv \pi V_{0} / \lambda_{s}$ (the peak frequency) as

$$
U\left(\omega_{0}\right)=\sum_{m=0}^{2 n-1} F_{0}\left(\omega_{0}\right)=2 n \cdot F_{0}\left(\omega_{0}\right)
$$

Thus, the reffectivity $R_{n}\left(\omega_{0}\right)$ of the medium defined as (40) and (41) can be expressed as

$$
R_{n}\left(\omega_{0}\right)=n \cdot R_{1}\left(\omega_{0}\right) .
$$

Utilizing the above relation, the theoretical value of $R_{n}\left(\omega_{0}\right)$ is computed and listed in Table 1 for sinusoidal structures in the case $\varepsilon=0.3$, and in Table 2 for periodic layered structures in the cases $s=0.2$ and $\varepsilon=0.02$. We find that the peak value obtained by the present theory is smaller than the real value, which is evaluated by the matrix method for the approximate layered model of the structure, when the length of the heterogeneous medium becomes great. Such difference arises from the first-order approximation, where the reflected wave does not diminish in the rear part as expected in reality.

\section{Attenuation of Elastic Waves Propagated through a Heterogeneous Medium}

Owing to reflection, the elastic waves in a heterogeneous medium attenuate during their propagation. The extent of attenuation is closely related to the reflectivity of the heterogeneous medium.

In the previous sections we find the characteristic of the reflectivity has a peak for $\lambda=2 \lambda_{3}$, where $\lambda$ is the wavelength of the incident wave, and $\lambda_{8}$ is that of the function $f(x)$; when the structure of the heterogeneous medium contains various components of wavelength of the fluctuation, the characteristic has many peaks corresponding to each component of wavelength. Then, the attenuation due to a given heterogeneous medium should be dependent on the wavelength or the frequency of an incident wave. To the first-order approximation, the wave with the wavelength $\lambda$ is only sensitive to the structure with the wavelength $\lambda / 2$.

Let us determine the equivalent attenuation factor $Q(\lambda)$ of a wave passing through a periodic structure as (40) and (41) where $\lambda=2 \lambda_{s}$. In determining the $Q$ value it is not necessarily assumed an exponential decay. The transmissivity $T_{n}$ and the reflectivity $R_{n}$ of the structure with the length $L\left(=n \lambda_{s}\right)$ satisfy the following relation.

$$
\left|T_{n}\right|=\sqrt{1-\left|R_{n}\right|^{2}}
$$

From (46) and (45), the transmissivity for $\lambda=2 \lambda_{3}$ is written as follows,

$$
\left|T_{n}(\lambda)\right|=\sqrt{1-n^{2}\left|R_{1}(\lambda)\right|^{2}},
$$

where $T_{n}$ and $R_{1}$ are taken as functions of the wavelength $\lambda$. On the other hand, 
the transmissivity is evaluated in terms of attenuation factor; then the following relation holds.

$$
e^{-\frac{\pi}{Q(n)} \cdot \frac{x}{\lambda}}=\left|T_{n}(\lambda)\right|
$$

When $n$ is a small number, we can rewrite (47) and (48) as follows:

$$
\begin{aligned}
& \left.\left|T_{n}\right|(\lambda)\left|\simeq 1-\frac{n^{2}}{2}\right| R_{1}(\lambda)\right|^{2} \\
& \mathrm{e}^{-\frac{\pi}{Q(\lambda)} \cdot \frac{L}{\lambda}}=e^{-\frac{\pi}{2 Q(\lambda)} \cdot n} \simeq 1-\frac{\pi}{2 Q(\lambda)} \cdot n .
\end{aligned}
$$

Combining the above relations, we get

$$
\frac{1}{Q(\lambda)}=\frac{n}{\pi}\left|R_{1}(\lambda)\right|^{2}
$$

provided $n\left|R_{1}(\lambda)\right|$ is small.

The relation (50) indicates that the decay of the amplitude is not exponential; the equivalent attenuation factor is dependent on the travel distance of the wave. YOSHIYAMA and ONDA (1962) and ONDA (1954) stated that the function of the attenuation is of hyperbolic secant. In the previous section it is found out that the amplitude of the real reflectivity $R_{n}$ is smaller than that of the present theory. Therefore the attenuation is over-estimated in (50). The true value of $Q(\lambda)$ is about in the following range as,

$$
\frac{1}{\pi}\left|R_{1}(\lambda)\right|^{2} \lesssim \frac{1}{Q(\lambda)}<\frac{n}{\mid \pi}\left|R_{1}(\lambda)\right|^{2} .
$$

In practical problems, the heterogeneity may be scattered in the medium; the heterogeneous structure with small length and certain wavelength is located separately in a homogeneous region or a heterogeneous medium with a different wavelength.

Let us divide the medium into parts where the particular structure is one by one involved; the length of the part is $L_{i}(i=1,2, \cdots, l)$, and the length of the hetorogeneous structure is $n_{i} \lambda_{8}\left(<L_{i}\right)$; the corresponding attenuation factor is $Q_{i}(\lambda)$, where $\lambda=2 \lambda_{s}$. The resultant attenuation factor $Q(\lambda)$ may be obtained as

$$
\frac{\sum_{i=1}^{l} L_{i}}{Q(\lambda)}=\sum_{i=1}^{l} \frac{L_{i}}{Q_{i}(\lambda)} .
$$

If $n$ is 1 or 2 , the following relation holds.

$$
\frac{1}{Q_{i}(\lambda)} \lesssim \frac{1}{\pi}\left|R_{1 i}(\lambda)\right|^{2}
$$

where $R_{1 i}(\lambda)$ means the reflectivity of the heterogeneous structure with one cycle in $i$-th part of the medium. Combining (52) and (53), we obtain $Q(\lambda)$ as follows,

$$
\frac{1}{Q(\lambda)} \lesssim \frac{1}{\pi \sum_{i=1}^{l} L_{i}} \cdot \sum_{i=1}^{l} L_{i}\left|R_{1 i}(\lambda)\right|^{2} \leq \frac{1}{\pi}\left|R_{1}(\lambda)\right|^{2},
$$


where $\left|R_{1}(\lambda)\right|$ is the maximum value of $\left|R_{1 i}(\lambda)\right|(i=1,2, \cdots, l)$.

If the structure is not continuously periodic, the above result may be of use in practical problems. In the case of a sinusoidal structure with one cycle, the reflectivity is easily calculated from (36) and we have

$$
\left|R_{1}(\lambda)\right|=\frac{\pi}{4} \varepsilon
$$

Then, the corresponding attenuation factor is eventually,

$$
\frac{1}{Q(\lambda)}=\frac{\pi}{4}\left(\frac{\varepsilon}{2}\right)^{2}, \quad \lambda=2 \lambda_{s} \text {. }
$$

If the periodic structure is thick enough, the equivalent attenuation factor discussed above is almost zero. This extreme case may not be encountered in practical problems.

\section{Finite Difference Method for Elastic Waves Propagated through a Heterogeneous Medium}

As one of the applications of the approximate theory, the numerical computation of the present problem is carried out by the use of finite difference method. The results will be also useful for understanding the effect of the heterogeneity on elastic waves.

The adopted structure of the medium in this numerical study is a sinusoidally fluctuated one. The partial differential equation and the medium structure are as follows:

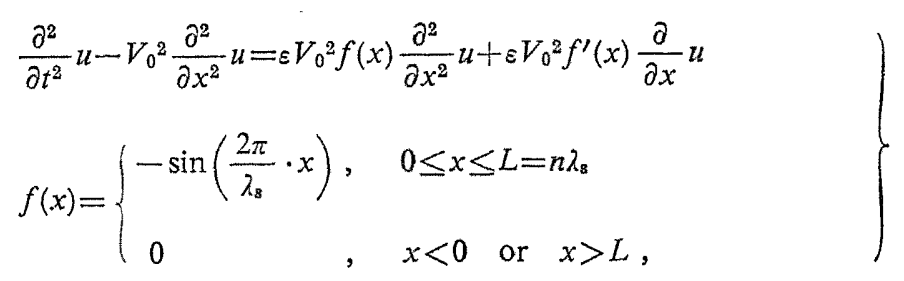

where $n=1 / 2,1,3 / 2, \cdots$.

It should be noted that discontinuities appear for $x=0$ and $x=L$ in $f^{\prime}(x)$. This situation is unfavorable for this finite difference approximation.

The difficulty is overcome by utilizing the result of Section 2. The contribution of the right-hand side of the wave equation to the reflected wave is shown in (16), (19) and (20). If we only notice the reflected wave, the following relation should hold.

$$
u_{1}=v_{1}+v_{2}=-v_{1} .
$$

Thereupon, taking account of (58), the wave equation is reduced to such a simple form as

$$
\frac{\partial^{2}}{\partial t^{2}} u-V_{0}^{2}(1-\varepsilon f(x)) \frac{\partial^{2}}{\partial x^{2}} u=0
$$



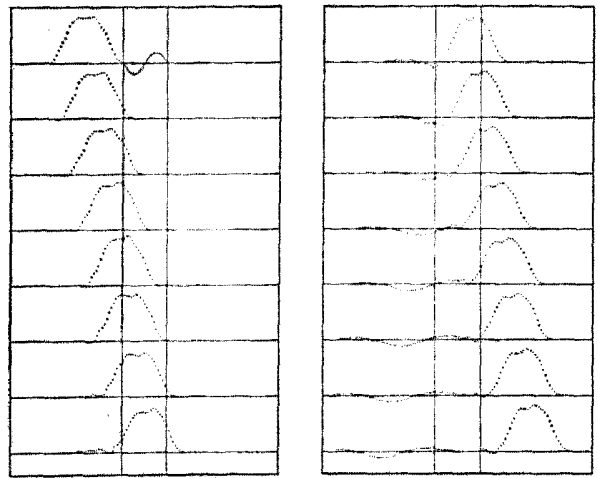

Fig. 7. Wave motion propagated through the sinusoidally fluctuated medium with one cycle obtained by the finite difference method.

The wave is propagated in the right direction in the figure as time lapses.

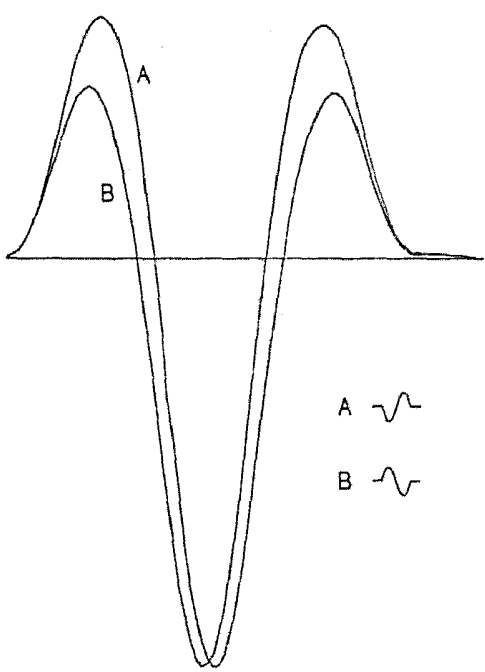

Fig. 9. (A) Reflected wave obtained by the matrix method against the incident wave shown in Fig. 7 or Fig. 8 for $\varepsilon=0.3$ and $f(x)=\left\{\begin{array}{cl}-\sin \left(\frac{2 \pi}{\lambda_{s}} \cdot x\right) & \left(0 \leq x<\lambda_{s}\right) \\ 0 & \left(x<0 \text { or } x>\lambda_{8}\right) .\end{array}\right.$

(B) Wave form equivalent to the reflected wave for $z=0.3$ and

$f(x)=\left\{\begin{array}{cl}\sin \left(\frac{2 \pi}{\lambda_{8}} \cdot x\right) & \left(0 \leq x \leq \lambda_{8}\right) \\ 0 & \left(x<0 \text { or } x>\lambda_{8}\right)\end{array}\right.$

except for its sign.
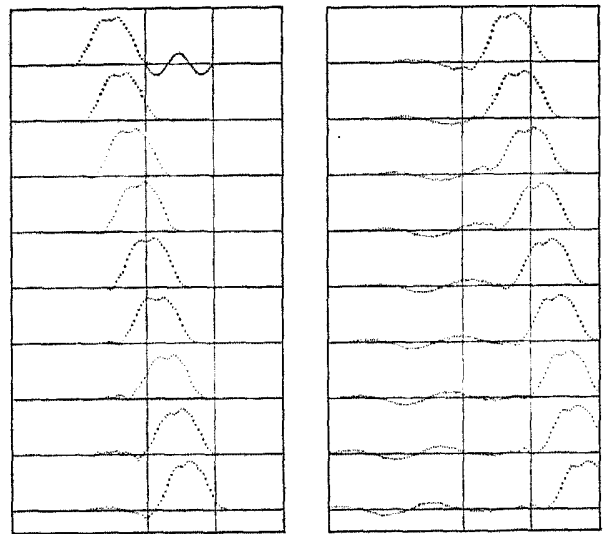

Fig. 8. Wave motion propagated through the sinusoidally fluctuated medium with one cycle and a half obtained by the finite difference method.

The wave is propagated in the right direction in the figure as time lapses.

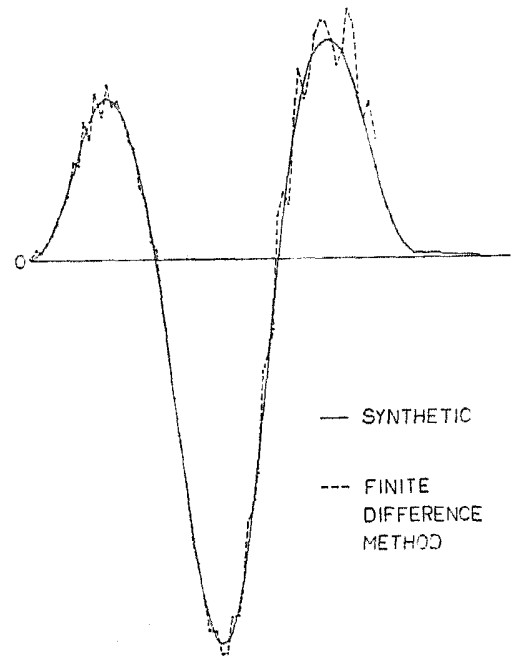

Fig. 10. Comparison between the refiected wave obtained by the finite difference method and (B) in Fig. 9.

Let Eq. (59) be transformed into the finite difference form as

$$
\begin{aligned}
& u_{j}^{n+1}-2 u_{j}^{n}+u_{j}^{n-1}=\left(\frac{\Delta t}{\Delta x} V_{0}\right)^{2} \\
& \quad \times\left(1-\varepsilon f\left(x_{j}\right)\right) \cdot\left[u_{j+1}^{n}-2 u_{j}^{n}+u_{j-1}^{n}\right],
\end{aligned}
$$

where $u_{j}^{n}$ means the solution at the time 
$t=t_{n}$ and at $x=x_{j} ; t_{n}$ and $x_{j}$ are the discrete time and space, respectively; $\Delta t$ and $\Delta x$ are lengths of intervals between any two grid points in space and time, respectively. The stability condition for homogeneous medium $(f(x)=0)$ is

$$
\alpha \equiv \frac{\Delta t}{\Delta x} \cdot V_{0}<1
$$

In the case of our problem, the numerical examination requires

$$
\alpha<0.5,
$$

for $\varepsilon=0.3$. The value of $\alpha$ is finally chosen to be 0.2 .

The incident wave is excited at the edge of the homogeneous medium.

The results are shown in Fig. 7 for $L=\lambda_{s}$, and in Fig. 8 for $L=1.5 \lambda_{s}$. The incident waves which are propagated in the right direction produce reflected waves while traveling through the heterogeneous medium. The wave forms of the reflected waves are in relation to the structure of the heterogeneous medium in such a manner that the prominent wave length of the reflected wave is twice that of the function of the structure. The figures also indicate as predicted by the theory that the effect of the heterogeneity on incident waves can be represented almost entirely by the characteristics of the reflected waves, that is, by the wave forms, the amplitude and the total length of the reflected wave trains.

The reflected wave synthesized by use of the incident wave shown in Fig. 7 or Fig. 8 and the reflectivity computed by the matrix method in the case $\varepsilon=0.3$ and (34) is given in Fig. 9 (A). In this figure (B) indicates the wave equivalent to the reflected wave except for its sign in the case $=0.3$ and

$$
f(x)= \begin{cases}\sin \left(\frac{2 \pi}{\lambda_{s}} \cdot x\right), & 0 \leq x \leq \lambda_{s} \\ 0 \quad, & x<0 \text { or } x>\lambda_{s} .\end{cases}
$$

It is found that the result of the finite difference method agrees well with (B) in Fig. 9 as shown in Fig. 10. By the present approximate theory the difference between (A) and (B) in Fig. 9 cannot be discernible. The extent of such difference must be decreased as the value $\varepsilon$ becomes smaller.

\section{Conclusion and Discussion}

An approximate solution of the elastic waves propagated through a heterogeneous medium is presented in this paper. The validity of the solution is confirmed by an examination of the theory.

The reflection is essential in considering the effect of the heterogeneity on elastic waves. The characteristic of the reflectivity is closely related to the structure of the heterogeneous medium. In so far as the first-order approximation is concerned, the inverse problem previously mentioned is solved by the present theory; the reflectivity is expressed with the function of the structure.

If the length of the heterogeneous medium is large or the function of the structure is not oscillatory, insufficiency of the approximation cannot be neglected. Never- 
theless, the first-order approximate solution has its own use; it shows gross aspect of the true solution. Even in such a case as the two homogeneous half spaces have different medium constants, the approximate reflected wave obtained by the theory describes the main feature of the true solution. For instance, in the case of the first-order discontinuity with two different homogeneous media, the reflectivity should be a flat function of frequency, which is also predicted by the present theory, although the theoretical amplitude is about one-half of the real value. In the case that the structure has linear transition layer the shape of the reflected wave is something like a rectangle, and its spectral amplitude is proportional to $f^{-1}$ ( $f$ :frequency) at high frequencies (see HIRASAWA and BERRY, 1971).

The property of reflected waves is often utilized in the study of crustal structure. The detailed structure of the Moho boundary is discussed by FucHs (1969), who insists that the band-limited (higher and lower frequency cut off) characteristic of the reflected waves from artificial earthquakes should indicate some periodic structure in the vicinity of the Moho plane. Utilizing the present theory, we can easily elucidate such a structure from the data of reflected waves.

In interpreting of reflected waves with band-limited spectra as above we can adopt sinusoidally fluctuated structures as standard models of structures of heterogeneities, for any structure can be considered as the superposition of sinusoidal components.

The reflection is namely the backward scattering of a wave. The forward scattering is, however, negligible to the first order approximation. Even in the three dimensional situation it is presumable that the scattered wave is intensive in the backward direction. YAMAKAWA (1962) investigated theoretically the nature of the scattering of elastic waves due to elastic spherical obstacles and found that the radiation patterns of the scattered waves have main lobes nearly in the direction from which an incident wave is propagated. Therefore, it can be said that the coda of seismic waves must mainly originate from the reflections of the initial waves, due to certain heterogeneities.

Concerning the attenuation due to the heterogeneity, we can say that, if the amplitude of the fluctuation of the heterogeneous medium is sufficiently small and the thickness of the medium is not so great, the equivalent attenuation factor is proportional to the travel distance of a propagating wave. That is, the waves in heterogeneous medium do not decay exponentially with range.

The finite difference method demonstrates the process by which the reflected waves are generated. The wave equation used in this method is reduced to a simple form with the aid of the present approximate theory.

The author wishes to thank Professor Ryosuke Sato and Mr. Yasunori Suzuki for discussions which are very helpful for examining the theoretical formulae obtained in the present study.

\section{REFERENCES}

Fuchs, K., Das Reflexions- und Transmissionsvermögen eines geschichteten Mediums mit beliebiger Tiefen-Verteilung der elastischen Moduln und der Dichte für schrägen Einfall ebener Wellen, Z. Geophys., 34, 389-413, 1968. 
Fuchs, K., On the properties of deep crustal reflectors, Z. f. Geophysik, 35, 133-149, 1969. HIRASAWA, T. and M. J. BERRY, Refiected and head waves from a linear transition layer in a fluid medium, Bull. Seism. Soc. Amer., 61, 1-25, 1971.

ONDA, I., Propagation and apparent attenuation of elastic waves in a heterogeneous medium with certain periodic structures, Bull. Earthq. Res. Inst., 42, 427-447, 1964.

ONDA, I., Effect of intervenient boundaries on the elastic wave propagation, Bull. Earthq. Res. Inst., 43, 1-14, 1965.

YAMAKAWA, N., Scattering and attenuation of elastic waves, Part I Scattering of elastic waves by various kinds of obstacles, Geophys. Mag., 31, 63-95, 1962.

YoshiYama, R., Stability of waves through a heterogeneous medium and apparent internal friction, Bull. Earthq. Res. Inst., 38, 467-478, 1960.

YoshiYama, R. and I. ONDA, Stability of waves through a heterogeneous medium and apparent internal friction, Part 2 with special remarks on wave propagation in a periodic structure, Bull. Earthq. Res. Inst., 40, 391-398, 1962. 